\title{
Efficacy of commercial formulas in comparison with home-made formulas for enteral feeding: A critical review
}

\author{
Majid Hassan-Ghomi ${ }^{1}$, Bahareh Nikooyeh ${ }^{2}$, Soudabeh Motamed ${ }^{2}$, Tirang R. Neyestani ${ }^{2 *}$ \\ Received: 12 Feb 2017 \\ Published: 5 Sep 2017
}

\begin{abstract}
Background: In several disease conditions, patients must inevitably be nourished by enteral feeding (EF). Though in many countries, commercial formulas are routinely used for EF, in Iran still home-made formulas are commonly employed as commercial formulas are not covered by insurance. This may pose patients to malnutrition and bring about further costs. The aim of this study was to evaluate the efficacy of EF commercial formulas in comparison with home-made formulas and thus to make further evidence for insurance policy-making

Methods: Medline, Cochrane, Embass and Center for Review \& Dissemination (CRD) as well as IranDoc and SID databases were searched. Keywords included formula, ICU, and enteral nutrition or tube feeding. No clinical trial study on the efficacy of EF formulas was found. Therefore, the compositions of available formulas and their cost-effectiveness were evaluated based on the clinical guidelines of scientific bodies such as American Society for Parenteral and Enteral Nutrition (ASPEN), European Society for Parenteral and Enteral Nutrition (ESPEN) and relative articles available in PubMed. In addition, the expert opinions were also taken into consideration.

Results: Domestic commercial formulas seemed to less merit dietary recommended intakes, i.e. the amount of some nutrients were much higher, and some others were much lower than the recommended values. The amount of several micronutrients including vitamins B1, B6, C, D and K, as well as iron, calcium and magnesium were not sufficient to meet the body needs in most commercial formulas upon receiving 2000 kilocalories and less.

Conclusion: Clinical studies on the efficacy of commercial formulas in comparison with home-made formulas are needed. Meanwhile, making suitable conditions for increasing the diversity of artificial nutrition products in the market would help clinical nutritionists to make better choices according to their patients conditions and to reduce the costs, as well.
\end{abstract}

Keywords: Enteral feeding, Enteral formula, Home-made formula, Intensive care unit

Copyright@ Iran University of Medical Sciences

Cite this article as: Hassan-Ghomi M, Nikooyeh B, Motamed S, Neyestani TR. Efficacy of commercial formulas in comparison with home-made formulas for enteral feeding: A review. Med J Islam Repub Iran. 2017 (5 Sep);31:55. https://doi.org/10.14196/mjiri.31.55

\section{Introduction}

Despite definition of "hospital malnutrition" in more than three decades ago, this problem still exists and is ignored somehow (1). Hospital malnutrition, particularly in the patients of intensive care unit (ICU) hospitalized for more than three days tends to be more severe, mostly due to the acute inflammatory response and consequent catabolic stress. This response is in conjunction with complications such as fatal infections, multi-organ dysfunction syndrome (MODS), and prolongation of hospitalization period and increase of mortality (2). Under these conditions, the circulating concentrations of water soluble vita-

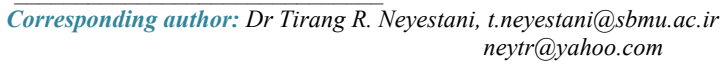

1. Department of Technology Assessments, Standard and Tariff for Health, Deputy for Healthcare Affairs, Ministry of Health and Medical Education, Tehran, Iran.

2. Laboratory of Nutrition Research, National Nutrition and Food Technology Research Institute; and Faculty of Nutrition Sciences and Food Technology, Shahid Beheshti University of Medical Sciences, Tehran, Iran. mins, some trace elements (including selenium, zinc and iron), protein transporters and many antioxidants (particularly vitamin C) may decrease while the blood levels of some other elements, notably copper and manganese, $\mathrm{m}$ ay increase (3).

Malnutrition among ICU patients is quite prevalent. There is no report of the occurrence rate of hospital malnutrition in Iran. However, the prevalence of this problem in the United States has been reported $40 \%$ in 1995 (4). In the critically ill patients, malnutrition is along with the impaired immune function, weakness of respiratory mus-

$\uparrow$ What is "already known" in this topic:

Commercial formulas are routinely used for enteral feeding. In Iran, still home-made formulas are commonly employed as commercial formulas are not covered by insurance.

\section{$\rightarrow$ What this article adds:}

The review of the literature revealed that the amount of several micronutrients including vitamins B1, B6, C, D and $\mathrm{K}$, as well as iron, calcium and magnesium were not sufficient to meet the body needs in most commercial formulas upon receiving 2000 kilocalories and less. 
cles, prolongation of dependence to artificial ventilation and increased mortality (5).

During the last three decades, the importance of molecular and biologic impacts of nutrients in the critical patients has been more appreciated. Studies indicate that plenty of ICU patients receive much less energy and protein than their requirements (averagely, $49-70 \%$ of the required value) $(6,7)$. In 1950 , it was proved that energyprotein malnutrition is associated with increased mortality and disease complications and provision of adequate energy and protein for critically ill patients may improve the clinical consequences (6).

Recent researches indicate that nutritional care is effective in reducing the morbidity and mortality in critically ill patients $(8,9)$. In several disease conditions, patients must inevitably be nourished by enteral feeding (EF), in which food stuffs and liquid are given to the patient in the form of a homogenous liquid named formula via a special tube inserted in patient's digestive tract.

During recent years, the diversity of EF formulas has been rapidly increased. Nowadays, there are several kinds of formulas in the market that occasionally may make the selection difficult. In most cases, a formula is selected based on the experience of physician or nutritionist, availability and finally the price. In other words, the effectiveness and indication thereof may be less taken into consideration. On the other hand, as Food and Drug Administration (FDA) considers formulas as nutritional supplements, they are not under legal supervision similar to the drugs. Therefore, the manufacturers may make many claims without supporting scientific evidence. The most common formulas available in the Iranian market include Entramil, Milatech, Ensure, Notricamp and Fresubin. Currently, number of imported formulas is very limited and even may not be easily available in the market. Though in many countries, commercial formulas are routinely used for EF, in Iran still home-made formulas are commonly employed as commercial formulas are not covered by insurance. This may pose patients to malnutrition and bring about further costs. This study, therefore, aimed to evaluate the efficacy of EF commercial formulas in comparison with home-made formulas and thus to make further evidence for insurance policy-making.

\section{Methods}

Medline, Cochrane, Embass and Center for Review \& Dissemination (CRD) as well as IranDoc and SID databases were searched. Keywords included "formula", "ICU", and "enteral feeding" or "enteral nutrition" or "tube feeding". No clinical trial study on the efficacy of EF formulas was found. Therefore, the composition of available formulas was investigated and their costeffectiveness was determined based on the clinical guides of scientific authorities notably American Society for Parenteral and Enteral Nutrition (ASPEN) $(2,10,11)$ and European Society for Parenteral and Enteral Nutrition (ESPEN) (12-16). Moreover the composition of all formulas available in the Iranian market was compared to the composition recommended by the competent authorities. Meanwhile, the composition of a home-made formula with a recipe recommended by the Iran Nutrition Society (INS) was investigated and its cost effectiveness was compared to that of commercial formulas available in the Iranian markets. In this study, the cost needed for feeding the patients was estimated based on current prices. The sufficiency of formulas for providing the required energy and nutrients was judged based on the Dietary Reference Intakes (DRIs).

\section{Results and discussion Effectiveness}

The commercial EF formulas can be categorized as standard, elemental and disease-specific. There are many formulas available in each category often with different compositions. Standard formulas are designed to meet the basic needs to protein together with a balanced value of other macronutrients and micronutrients. These formulas have lower price than special formulas. Special (diseasespecific) formulas are designed for specific clinical conditions (Table 1) (2). Selection of an appropriate formula for $\mathrm{EF}$ is mostly dependent on the following factors: nutritional needs of the patient, function of the patient's digestive system, location of the tube end (stomach or intestine), food sensitivities and/or lactose intolerance in the

Table 1. Classification of enteral formulas

\begin{tabular}{|c|c|c|c|}
\hline Class & Subgroup & Specifications & Indication \\
\hline & Standard & Similar to normal diet & Normal digestion \\
\hline & High protein & Protein more than $15 \%$ of total calorie & $\begin{array}{l}\text { - Catabolism } \\
\text { - Wound healing }\end{array}$ \\
\hline Polymeric & High energy & More than 1 kilocalorie per $\mathrm{ml}$ & - Constraint of liquids \\
\hline & & & $\begin{array}{l}\text { - Intoleration of high volume } \\
\text { - Abnormality of electrolytes }\end{array}$ \\
\hline & Fibrous & $5-15$ gr of fiber per lit & - Adjustment of intestine function \\
\hline & Partial hydrolysis & One or more nutrients are digested. & Problem of digestion and absorption \\
\hline Monomeric & $\begin{array}{l}\text { Elemental } \\
\text { Peptide }\end{array}$ & & \\
\hline \multirow{6}{*}{$\begin{array}{l}\text { For special } \\
\text { diseases }\end{array}$} & Renal & Low protein and electrolyte & Renal disorder \\
\hline & Hepatic & High BCAAs, low FAAs and electrolytes & Hepatic encephalopathy \\
\hline & Pulmonary & High fat & ARDS \\
\hline & Diabetic & Low carbohydrate & Diabetes \\
\hline & Safety increaser & Arginine, glutamine, omega 3 , and antioxidant & - Metabolic problem \\
\hline & & & - Safety system problem \\
\hline
\end{tabular}

Abbreviations: ARDS: acute respiratory distress syndrome; BCAAs: branched chain amino acids; FAAs: free amino acids 
patient, and the amounts of sodium, potassium, magnesium and phosphorous available in the formula, particularity in the patients suffering from renal, hepatic or cardiacrespiratory failure. To our knowledge, there is no study ever having investigated the effectiveness of formulas available in the Iranian market and other countries. Thus, attempts were made to evaluate and compare the composition and cost-effectiveness of the home-made and commercial formulas available in Iranian market based on recent recommendations of scientific authorities.

\section{Nutrient composition of the enteral formulas}

Energy, micronutrients and macronutrients available in $100 \mathrm{~mL}$ of each formula against the ASPEN recommended values are shown in Table 2. Standard formulas majorly contain micro- and macro-nutrients to the extent of values recommended for the healthy persons. The ASPEN guideline (2) has modified the amounts of nutrients required for people under enteral and parenteral nutrition. However, more amounts of energy and nutrients may be needed to meet critically ill patients' requirements (2).

\section{Macronutrients}

Most commercial formulas used in EF contain 1 $\mathrm{kcal} / \mathrm{mL}$. A few provide $2 \mathrm{kcal} / \mathrm{mL}$ that may be used for the patients with intensive limitation for liquid intake (such as patients suffering from cardiac, respiratory, renal or hepatic failures).

In most commercial formulas, the ratio of energy from non-protein nutrients to nitrogen is $150 \mathrm{kcal} / \mathrm{g}$. This ratio is ideal for the patients without stress. In patients with increased need to protein (e.g. those suffering from burning, infection or multiple traumas), use of commercial protein mixtures or commercial high protein formulas is recommended. Based on the ASPEN guideline, protein needs for these patients is $0.8-2.0 \mathrm{~g} / \mathrm{kg} . \mathrm{d}^{-1}, 25-30 \%$ of which must be provided from essential amino acids (10, 11). This value is about $15-25 \%$ of daily energy intake. The recommended ratio of non-protein calorie to nitrogen is about 70:1 to 100:1 (2). The protein in polymeric formulas is typically derived from casein, casemate, whey protein, egg (ovo-albumin), soya (soy protein), sodium, potassium, calcium and magnesium caseinates, lactalbumin, and milk protein extract. Almost all formulas except elemental or predigested ones that have protein as di or tripepetides and amino acids, provide intact protein and are gluten free (5).

The amount of protein in polymeric formulas available in the domestic commercial products varies from $15 \%$ to $20 \%$ of total energy, with high protein formulas having the highest amount of protein among these formulas. If $1 \mathrm{~g} / \mathrm{kg} . \mathrm{BW}^{-1}$ protein is prescribed for a $70 \mathrm{~kg}$ man, then he must receive at least $1870 \mathrm{ml}$ of a standard formula containing $15 \%$ of total energy from protein to supply his needed protein.

Fat sources in polymeric formulas are corn oil, sunflower oil with high oleate, soy, flax seed oil, soy lecithin, mono- and di-glyceride, canola oil, fish oil, date seed oil,

Table 2. Nutrient composition of Entramil formulas in comparison with the daily intake values (DV\%) recommended by ASPEN

\begin{tabular}{|c|c|c|c|c|c|c|c|c|c|c|}
\hline & Unit & $\begin{array}{l}\text { Standard } \\
\text { Entramil }\end{array}$ & DV\% & $\begin{array}{l}\text { Diabetic } \\
\text { Entramil }\end{array}$ & DV\% & $\begin{array}{c}\text { High protein } \\
\text { Entramil }\end{array}$ & DV\% & $\begin{array}{l}\text { Fibrous } \\
\text { Entramil }\end{array}$ & $\mathrm{DV} \%$ & $\begin{array}{c}\text { ASPEN } \\
\text { DRI }\end{array}$ \\
\hline Formula shape & - & Powder & - & Powder & - & Powder & - & Powder & - & - \\
\hline Energy & Kilocalorie & 100 & - & 100 & - & 120 & - & 100 & - & - \\
\hline Protein & Percent & 14 & - & 15 & - & 17 & - & 15.4 & - & - \\
\hline Carbohydrate & Percent & 54.4 & - & 37 & - & 48 & - & 52 & - & - \\
\hline Fat & Percent & 31.6 & - & 48 & - & 35 & - & 32.6 & - & - \\
\hline Fiber & Percent & 0.4 & - & 0.98 & - & 0.5 & - & 1.36 & - & - \\
\hline Osmolality & Miliosmol & 340 & - & 380 & - & 380 & - & 380 & - & - \\
\hline B1 & Milligram & 0.15 & - & 0.18 & 300 & 0.18 & 250 & 0.16 & 266 & 1.2 \\
\hline B2 & Milligram & 0.17 & 250 & 0.2 & 307 & 0.2 & 256 & 0.17 & 261 & 1.3 \\
\hline Niacin & Milligram & 1.27 & 261 & 1.89 & 236 & 2.23 & 232 & 1.31 & 163 & 16 \\
\hline Pantothenic acid & Milligram & 0.63 & 158 & 0.69 & 276 & 0.75 & 250 & 0.65 & 260 & 5 \\
\hline B6 & Milligram & 0.13 & 252 & 0.14 & 164 & 0.15 & 147 & 0.13 & 152 & 1.7 \\
\hline Folic acid & Microgram & 25.4 & 152 & 27.28 & 136 & 30 & 125 & 26.3 & 131 & 400 \\
\hline Biotin & Microgram & 15.85 & 127 & 17.05 & 1136 & 18.76 & 1042 & 16.45 & 1096 & 30 \\
\hline B12 & Microgram & 0.5 & 1056 & 0.55 & 458 & 0.59 & 409 & 0.52 & 433 & 2.4 \\
\hline Vitamin C & Milligram & 9.72 & 416 & 10.2 & 226 & 11.25 & 208 & 9.86 & 219 & 90 \\
\hline Vitamin D & IU & 19 & 216 & 20.47 & 68 & 22.5 & 62 & 19.75 & 65 & 600 \\
\hline Vitamin E & IU & 2.5 & 63 & 2.73 & 242 & 2.99 & 221 & 2.62 & 232 & 22.5 \\
\hline Vitamin K & Microgram & 6.34 & 222 & 6.8 & 113 & 7.48 & 103 & 6.56 & 109 & 120 \\
\hline Vitamin A & IU & 209.5 & 105 & 225.2 & 150 & 247.56 & 137 & 217 & 144 & 3000 \\
\hline Iron & Milligram & 1.53 & 139 & 1.12 & 124 & 1.11 & 102 & 0.98 & 108 & 18 \\
\hline Calcium & Milligram & 77.2 & 170 & 73.67 & 122 & 82.58 & 114 & 72.4 & 120 & 1200 \\
\hline Chrome & Milligram & 1.85 & 128 & 2.47 & 164 & 2.24 & 124 & 1.97 & 131 & 30 \\
\hline Copper & Milligram & 0.09 & 123 & 0.1 & 222 & 0.1 & 185 & 0.09 & 200 & 0.9 \\
\hline Fluorine & Milligram & 0.22 & 200 & 0.23 & 115 & 0.25 & 104 & 0.22 & 110 & 4 \\
\hline Iodine & Milligram & 9.5 & 110 & 10.23 & 136 & 11.25 & 125 & 9.86 & 131 & 150 \\
\hline Magnesium & Milligram & 37.4 & 126 & 25.05 & 119 & 27.92 & 110 & 24.48 & 116 & 420 \\
\hline Manganese & Milligram & 0.1 & 178 & 0.18 & 156 & 0.18 & 130 & 0.16 & 139 & 2.3 \\
\hline Molybdenum & Microgram & 2.85 & 86 & 3.06 & 136 & 3.38 & 125 & 2.96 & 131 & 45 \\
\hline Zinc & Milligram & 1.2 & 126 & 0.87 & 158 & 1.11 & 168 & 0.98 & 178 & 11 \\
\hline Phosphorous & Milligram & 77.2 & 218 & 43.7 & 124 & 80 & 190 & 72.4 & 206 & 700 \\
\hline Potassium & Milligram & 92 & 220 & 147.5 & 147 & 192 & 160 & 162.9 & 162 & 2000 \\
\hline Sodium & Milligram & 52.6 & 92 & 139.1 & 556 & 144 & 480 & 122.17 & 488 & 500 \\
\hline Chlorine & Milligram & 83 & 210 & 75.83 & 202 & 92.9 & 206 & 70.1 & 186 & 750 \\
\hline Selenium & Microgram & 3.5 & 221 & 3.75 & 136 & 4.13 & 125 & 3.62 & 131 & 55 \\
\hline
\end{tabular}


Table 3. Nutrient composition of Milatech formulas in comparison with the recommended daily intake values (DV\%)

\begin{tabular}{|c|c|c|c|c|c|c|c|c|c|c|c|c|c|c|c|c|c|c|}
\hline & Unit & $\begin{array}{l}\text { Standard } \\
\text { Milatech }\end{array}$ & DV\% & $\begin{array}{c}\text { Standard } \\
\text { fibrous } \\
\text { Milatech }\end{array}$ & DV\% & $\begin{array}{c}\text { High } \\
\text { protein } \\
\text { Milatech }\end{array}$ & DV\% & $\begin{array}{c}\text { High } \\
\text { energy } \\
\text { Milatech }\end{array}$ & DV\% & $\begin{array}{l}\text { Intensive } \\
\text { Milatech }\end{array}$ & DV\% & $\begin{array}{l}\text { Elemental } \\
\text { Milatech }\end{array}$ & DV\% & $\begin{array}{c}\text { Safety } \\
\text { Milatech }\end{array}$ & DV\% & $\begin{array}{l}\text { Pehatic } \\
\text { Milatch }\end{array}$ & DV\% & $\begin{array}{c}\text { ASPEN } \\
\text { DRI }\end{array}$ \\
\hline $\begin{array}{l}\text { Formula } \\
\text { shape }\end{array}$ & - & Solution & - & Solution & - & Solution & - & Solution & - & Solution & - & Solution & - & Solution & - & Solution & - & - \\
\hline Energy & Kilocalorie & 100 & - & 100 & - & 100 & - & - & - & 130 & - & 100 & - & 133 & - & - & - & - \\
\hline High protein & Percent & 15 & - & 15 & - & 20 & - & - & - & 20 & - & 20 & - & 20 & - & - & - & - \\
\hline Carbohydrate & Percent & 55 & - & 55 & - & 50 & - & - & - & 40 & - & 70 & - & 55 & - & - & - & - \\
\hline Fat & Percent & 30 & - & 30 & - & 30 & - & - & - & 40 & - & 10 & - & 25 & - & - & - & - \\
\hline Fiber & gr & 0 & - & 1.5 & - & 0 & - & - & - & 0 & - & 0 & - & 0 & - & - & - & - \\
\hline Osmolarity & Miliosmol & - & - & - & - & - & - & - & - & - & - & - & - & - & - & - & - & - \\
\hline B1 & Milligram & 0.07 & 116 & 0.07 & 116 & 0.07 & 116 & 116 & 288 & 0.07 & 107 & 0.07 & 116 & 0.14 & 175 & 175 & 153 & 1.2 \\
\hline B2 & Milligram & 0.08 & 123 & 0.08 & 123 & 0.08 & 123 & 123 & 307 & 0.08 & 94 & 0.08 & 123 & 0.16 & 185 & 185 & 177 & 1.3 \\
\hline Niacin & Milligram & 1 & 125 & 1 & 125 & 1 & 125 & 125 & 250 & 1.3 & 125 & 1 & 125 & 1.62 & 152 & 152 & 159 & 16 \\
\hline $\begin{array}{l}\text { Pantothenic } \\
\text { acid }\end{array}$ & Milligram & 0.2 & 80 & 0.2 & 80 & 0.2 & 80 & 80 & 80 & 0.2 & 61 & 0.2 & 80 & 0.33 & 99 & 99 & 92 & 5 \\
\hline B6 & Milligram & 0.11 & 129 & 0.11 & 129 & 0.11 & 129 & 129 & 235 & 0.12 & 108 & 0.11 & 129 & 0.24 & 212 & 212 & 162 & 1.7 \\
\hline Folic acid & Milligram & 20 & 100 & 20 & 100 & 20 & 100 & 100 & 150 & 20 & 76 & 20 & 100 & 30 & 112 & 112 & 115 & 400 \\
\hline Biotin & Microgram & 8 & 533 & 8 & 533 & 8 & 533 & 533 & 355 & 8 & 410 & 8 & 533 & 8 & 401 & 401 & 410 & 30 \\
\hline B12 & Microgram & 0.15 & 125 & 0.15 & 125 & 0.15 & 125 & 125 & 150 & 0.15 & 96 & 0.15 & 125 & 0.36 & 225 & 225 & 96 & 2.4 \\
\hline Vitamin C & Milligram & 3 & 66 & 3 & 66 & 3 & 66 & 66 & 296 & 3 & 51 & 3 & 66 & 6 & 100 & 100 & 102 & 90 \\
\hline Vitamin D & IU & 20 & 66 & 20 & 66 & 20 & 66 & 66 & 133 & 26 & 66 & 20 & 66 & 33.6 & 84 & 84 & 66 & 600 \\
\hline Vitamin E & IU & 0.75 & 66 & 0.75 & 66 & 0.75 & 66 & 66 & 331 & 0.75 & 51 & 0.75 & 66 & 1.9 & 126 & 126 & 102 & 22.5 \\
\hline Vitamin $\mathrm{K}$ & Microgram & 3.5 & 58 & 3.5 & 58 & 3.5 & 58 & 58 & 116 & 4.55 & 58 & 3.5 & 58 & 5.33 & 66 & 66 & 76 & 120 \\
\hline Vitamin A & IU & 150 & 100 & 150 & 100 & 150 & 100 & 100 & 177 & 150 & 76 & 150 & 100 & 333 & 166 & 166 & 93 & 3000 \\
\hline Iron & Milligram & 0.9 & 100 & 0.9 & 100 & 0.9 & 100 & 100 & 148 & 0.9 & 76 & 0.9 & 100 & 1.57 & 131 & 131 & 115 & 18 \\
\hline Calcium & Milligram & 45 & 75 & 45 & 75 & 45 & 75 & 75 & 112 & 54 & 69 & 45 & 75 & 60 & 75 & 75 & 69 & 1200 \\
\hline Chrome & Microgram & - & & - & & - & & & & - & & - & & - & & & & 30 \\
\hline Copper & Milligram & 0.1 & 222 & 0.1 & 222 & 0.1 & 222 & 222 & 222 & 0.1 & 170 & 0.1 & 222 & 0.16 & 267 & 267 & 273 & 0.9 \\
\hline Fluorine & Milligram & 0.1 & 50 & 0.1 & 50 & 0.1 & 50 & 50 & 50 & 0.1 & 38 & 0.1 & 50 & 0.16 & 60 & 60 & 76 & 4 \\
\hline Iodine & Microgram & 7.5 & 100 & 7.5 & 100 & 7.5 & 100 & 100 & 128 & 7.5 & 76 & 7.5 & 100 & 12 & 120 & 120 & 115 & 150 \\
\hline Magnesium & Milligram & 17.5 & 83 & 17.5 & 83 & 17.5 & 83 & 83 & 111 & 18.5 & 67 & 17.5 & 83 & 30.87 & 110 & 110 & 105 & 420 \\
\hline Manganese & Milligram & 0.12 & 104 & 0.12 & 104 & 0.12 & 104 & 104 & 197 & 0.12 & 80 & 0.12 & 104 & 0.22 & 147 & 147 & 160 & 2.3 \\
\hline Molybdenum & Microgram & 5 & 222 & 5 & 222 & 5 & 222 & 222 & 222 & 5 & 170 & 5 & 222 & 6.5 & 217 & 217 & 222 & 45 \\
\hline Zinc & Milligram & 0.75 & 136 & 0.75 & 136 & 0.75 & 136 & 136 & 254 & 0.75 & 104 & 0.75 & 136 & 0.94 & 128 & 128 & 163 & 11 \\
\hline Phosphorous & Milligram & 40 & 114 & 40 & 114 & 40 & 114 & 114 & 185 & 60 & 131 & 40 & 114 & 66.6 & 143 & 143 & 131 & 700 \\
\hline Potassium & Milligram & 130 & 130 & 130 & 130 & 130 & 130 & 130 & 86 & 130 & 100 & 130 & 130 & 130 & 97 & 97 & 100 & 2000 \\
\hline Sodium & Milligram & 50 & 200 & 50 & 200 & 50 & 200 & 200 & 133 & 50 & 153 & 50 & 200 & 50 & 150 & 150 & 153 & 500 \\
\hline Chlorine & Milligram & 95 & 253 & 95 & 253 & 95 & 253 & 253 & 266 & 105 & 215 & 95 & 253 & 146.6 & 293 & 293 & 287 & 750 \\
\hline Selenium & Microgram & 2.5 & 90 & 2.5 & 90 & 2.5 & 90 & 90 & 164 & 3.5 & 97 & 2.5 & 90 & 3.6 & 98 & 98 & 97 & 55 \\
\hline
\end{tabular}




\begin{tabular}{|c|c|c|c|c|c|c|c|c|c|c|c|c|}
\hline & Unit & Ensure & $D V \%$ & $\begin{array}{c}\text { Standard } \\
\text { Nutricamp }\end{array}$ & $D V \%$ & $\begin{array}{c}\text { High energy } \\
\text { Nutricamp }\end{array}$ & $D V \%$ & $\begin{array}{l}\text { Standard } \\
\text { Fresubin }\end{array}$ & $D V \%$ & $\begin{array}{c}\text { High protein } \\
\text { Fresubin }\end{array}$ & $D V \%$ & $\begin{array}{c}\text { ASPEN } \\
D R I\end{array}$ \\
\hline Formula shape & & Powder & - & Solution & - & Solution & - & Solution & - & Solution & - & - \\
\hline Energy & Kilocalorie & 100 & - & 100 & - & 150 & - & 100 & - & 150 & - & - \\
\hline Protein & Percent & 14 & - & 15 & - & 20 & - & 15 & - & 20 & - & - \\
\hline Carbohydrate & Percent & 54 & - & 55 & - & 50 & - & 55 & - & 45 & - & - \\
\hline Fat & Percent & 32 & - & 30 & - & 30 & - & 30 & - & 35 & - & - \\
\hline Fiber & $g r$ & 0.84 & - & 0 & - & 0 & - & 0 & - & 0 & - & - \\
\hline Osmolarity & Miliosmol & 395 & - & 250 & - & 344 & - & 250 & - & 300 & - & - \\
\hline$B 1$ & Milligram & 0.17 & 283 & 0.2 & 333 & 0.2 & 222 & 0.13 & 216 & 0.01 & 88 & 1.2 \\
\hline Niacin & Milligram & 2.3 & 287 & 1.8 & 225 & 1.8 & 150 & 1.6 & 200 & 1.06 & 88 & 16 \\
\hline Pantothenic acid & Milligram & 1.2 & 480 & 0.6 & 240 & 0.6 & 160 & 0.47 & 188 & 0.31 & 82 & 5 \\
\hline$B 6$ & Milligram & 0.23 & 270 & 0.2 & 235 & 0.2 & 156 & 0.16 & 188 & 0.1 & 78 & 1.7 \\
\hline Folic acid & Milligram & 46 & 230 & 30 & 150 & 30 & 100 & 27 & 135 & 18 & 60 & 400 \\
\hline Biotin & Microgram & 35 & 2333 & 5 & 333 & 5 & 222 & 5 & 333 & 3.33 & 148 & 30 \\
\hline$B 12$ & Microgram & 0.72 & 600 & 0.3 & 250 & 0.3 & 166 & 0.27 & 225 & 0.18 & 100 & 2.4 \\
\hline Vitamin $C$ & Milligram & 16 & 355 & 4 & 88 & 20 & 296 & 6.67 & 148 & 4.46 & 66 & 90 \\
\hline Vitamin D & $I U$ & 22 & 73 & 40 & 133 & 40 & 88 & 40 & 133 & 26.4 & 58 & 600 \\
\hline Vitamin $E$ & $I U$ & 2.6 & 231 & 1.55 & 137 & 3 & 177 & 1.99 & 176 & 5.6 & 331 & 22.5 \\
\hline Vitamin $K$ & Microgram & 4.2 & 70 & 7 & 116 & 7 & 77 & 6.67 & 111 & 4.44 & 49 & 120 \\
\hline Calcium & Milligram & 65 & 108 & 75 & 125 & 75 & 83 & 80 & 133 & 53.33 & 59 & 1200 \\
\hline Chrome & Microgram & 4.6 & 306 & 7 & 466 & 7 & 311 & 6.7 & 446 & 4.46 & 198 & 30 \\
\hline Copper & Milligram & 0.12 & 266 & 0.15 & 333 & 0.15 & 222 & 0.13 & 288 & 0.08 & 118 & 0.9 \\
\hline Fluorine & Milligram & 0 & - & 0.1 & 50 & 0.1 & 33 & 0.13 & 65 & 0.08 & 26 & 4 \\
\hline Iodine & Microgram & 7.9 & 105 & 13 & 173 & 13 & 115 & 13.3 & 177 & 8.86 & 78 & 150 \\
\hline Magnesium & Milligram & 21 & 100 & 20 & 95 & 20 & 63 & 25 & 119 & 18 & 57 & 420 \\
\hline Manganese & Milligram & 0.28 & 243 & 0.2 & 173 & 0.2 & 115 & 0.27 & 234 & 0.18 & 104 & 2.3 \\
\hline Molybdenum & Microgram & 8.8 & 391 & 10 & 444 & 10 & 296 & 10 & 444 & 6.66 & 197 & 45 \\
\hline Zinc & Milligram & 1.3 & 236 & 1.2 & 218 & 1.5 & 181 & 1.2 & 218 & 0.8 & 96 & 11 \\
\hline Phosphorous & Milligram & 53 & 151 & 65 & 185 & 65 & 123 & 63 & 180 & 42 & 80 & 700 \\
\hline Potassium & Milligram & 155 & 155 & 150 & 150 & 150 & 100 & 125 & 125 & 156 & 104 & 2000 \\
\hline Sodium & Milligram & 84 & 336 & 100 & 400 & 100 & 266 & 75 & 300 & 80 & 213 & 500 \\
\hline Chlorine & Milligram & 142 & 378 & 100 & 266 & 100 & 177 & 115 & 306 & 122 & 216 & 750 \\
\hline Selenium & Microgram & 4.4 & 160 & 7 & 254 & 9 & 218 & 6.7 & 243 & 4.46 & 108 & 55 \\
\hline
\end{tabular}


Table 5. A sample of a home-made formula* (28)

\begin{tabular}{|c|c|c|c|c|c|c|c|c|c|c|c|}
\hline Food composition & Value (g or $\mathrm{ml}$ ) & Energy & Carbohydrate & Protein & Fat & Iron & Zinc & Calcium & Vitamin D (IU) & Vitamin A (IU) & Vitamin C (mg) \\
\hline $\begin{array}{l}\text { Meat (cooked and water- } \\
\text { removed) }\end{array}$ & 210 & 403 & - & 49 & 35 & 35 & 8.8 & 17 & --- & ---- & --- \\
\hline $\begin{array}{l}\text { Vegetable (cooked and wa- } \\
\text { ter-removed) }\end{array}$ & 600 & 294 & 30 & 12 & - & - & 2.94 & 150 & --- & 25662 & 19.2 \\
\hline $\begin{array}{l}\text { Fruit (cooked and water- } \\
\text { removed) }\end{array}$ & 600 & 348 & 90 & - & - & - & 0.42 & 42 & --- & 2574 & 15 \\
\hline Milk powder & 300 & 1074 & 30 & 20 & 15 & 15 & 13.23 & 3693 & --- & 45 & 16.8 \\
\hline Pasteurized milk & 720 & 403 & 36 & 24 & 15 & 15 & 2.95 & 1030 & --- & 540 & 7.9 \\
\hline Vegetable oil & 30 & 265 & - & - & 30 & 30 & --- & --- & --- & --- & --- \\
\hline Water & As Required & & --- & --- & --- & --- & --- & --- & --- & --- & --- \\
\hline Total & - & 2787 & 186 & 105 & 95 & 95 & 28.34 & 4932 & --- & 28821 & 58.9 \\
\hline
\end{tabular}

patient.

borage oil, and meat butter or fat; they are majorly enriched with medium chain triglycerides (MCTs) (17). Use of products containing fish oil and borage oil may reduce the need to artificial ventilation and hospitalization period in ICU (5). A few researches indicated that formulas containing MUFAs have been effective in the control of hyperglycemia after feeding without increasing insulin secretion (18).

According to ASPEN, total intake of lipid should not exceed from $2.5 \mathrm{~g} / \mathrm{kg} . \mathrm{d}^{-1}$, and $1-2 \%$ of daily energy intake should be provided by linoleic acid (omega 6 ) and $0.5 \%$ by alpha linolenic acid (omega 3 ) (19). Approximately $31-40 \%$ of total energy of formulas available in the market are provided by lipids. Nonetheless, fat content of available formulas is not specified on the labels.

Maltodextrin, corn syrup, hydrolyzed starch, sucrose, fructose and sugar alcohols are usually the carbohydrates found in polymeric formulas (17). ASEPN recommends that carbohydrate intake should not exceed $7 \mathrm{~g} / \mathrm{kg} \cdot \mathrm{d}^{-1}(2)$. The percent of total energy provided by carbohydrate in formulas available in Iran varies from $40 \%$ to $55 \%$.

It has been shown that soluble fibers may reduce diarrhea episodes in patients but no significant effect on duration of artificial ventilation and hospitalization has been reported yet (2). The increase of viscosity due to soluble fibers must be taken into account in making formulas (17). Currently, progress of technology has extremely solved this problem. The results obtained from the assessment of enteral formulas containing fiber for diarrhea control are contradictory. It is chiefly due to the use of all types of fibers. Fibers are classified based on their solubility in water. Soluble fibers such as pectin and gum, by absorption of water and sodium, may be effective in treatment of EF-related diarrhea. Insoluble fibers, on the other hand, increase the weight of feces and reduce the intestinal transit time (17). Some researchers have shown that the insoluble fibers may not reduce the occurrence of diarrhea. Besides, a few cases of intestinal obstruction have been reported in patients received insoluble fibers $(2,20)$. One study compared the effect of enteral formula with and without soluble fiber and concluded that diarrhea episodes were significantly decreased in the patients received fiber containing formula (21). Recently, fructo-oligosaccharide (FOS) is used in formulas as it is fermented quickly by colon bacteria and produces short chain fatty acids (SCFAs) that affect the bowel function and digestive health via several ways. These fatty acids serve as fuel for colon cells, and enhance intestinal mucus and water and sodium absorption (22). Fibrous formulas are closer to a regular diet. Notwithstanding, the evidence for their use is not adequate. It should be noted that fibrous formulas should not be given to the patients at risk of intestinal obstruction or ischemia.

\section{Micronutrients}

Upon daily intake of $1300-1900 \mathrm{ml}$ of commercial formulas, the needs to vitamins and minerals in most patients are met. In contrast, in patients with fluid restriction, vitamins and minerals supplementation may be necessary. 
Formulas supplemented with antioxidants (such as vitamin E and ascorbic acid) and minerals (like selenium, copper and zinc) have been demonstrated to favorably affect the outcomes of diseases (particularly in burns and traumas) and to reduce the period of ventilation in artificially ventilated patients (2). Based on the Canadian clinical guideline, use of antioxidants is useful for the patients suffering from acute respiratory distress syndrome (5).

Use of antioxidant nutrients may reduce mortality and days of artificial ventilation though may not affect the infectious complications in these patients (5). In a clinical study, intake of a fortified formula containing $67 \mu \mathrm{g} / \mathrm{dL}$ vitamin A $\left(\mathrm{DV} \%{ }^{l}=148\right), 13.3 \mathrm{mg} / \mathrm{dL}$ vitamin $\mathrm{C}$ $(\mathrm{DV} \%=295)$ and $4.98 \mathrm{mg} / \mathrm{dL}$ vitamin $\mathrm{E}(\mathrm{DV} \%=658)$ compared with isocaloric and isonitrogenic formulas resulted in improvement of antioxidant defense but did not affect the mortality, infection rate and hospitalization period (23).

Overall, trace element and vitamin supplementation improves the antioxidant capacity in the critically ill patients (24). As selenium and vitamins $\mathrm{E}$ and $\mathrm{C}$ contribute in recycling of other water- and fat-soluble antioxidants, using a mixture of antioxidant micronutrients is preferred to the single antioxidant supplementation (25).

Currently, the commonly available formula products in the market are Entramil and Milatech, DV\% of both of which is $65 \%$ for vitamin D. By intake of lower than 3000 kcal, none of them can supply the required value of this vitamin. DV\% for vitamins $\mathrm{E}$ and $\mathrm{K}$ are $66 \%$ and $58 \%$, respectively. The concentration of vitamin $\mathrm{C}$ and fluorine of Nutricamp formula is lower than the recommended value, and upon receiving less than 4000 kilocalories, the recommended value of fluorine is not provided. The concentration of micronutrients $\mathrm{B} 1$, niacin, pantothenic acid, B6, folic acid, biotin, vitamins $\mathrm{C}, \mathrm{D}$ and $\mathrm{K}$, calcium, fluorine, iodine, magnesium, phosphorous of high protein Fresubin is not supplied by receiving 2000 kilocalories and less. As for other micronutrients, the composition of the commercial products is almost similar (Tables 3 and 4).

\section{Comparison of cost-effectiveness}

There are few studies (and no study in Iran) on the costeffectiveness of commercial formulas as compared to the home-made formulas. In a clinical trial on 82 patients with intestinal fistula, home-made formula was compared versus hospital (commercial) formula. The results showed a shorter hospital stay in home EF group with a significantly lower costs and increased quality of life. However, there were no significant difference in duration of EF and the incidence of complications between two groups (26). Another study reported that implementation of home enteral nutrition improved clinical outcomes and decreased health care costs through weight gain in patients, reduced incidence of infectious complications and the cost of hospitalization (27). However, the amount of nutrient intakes from home-made formulas should always be taken into consideration as the amount of some micronutrients may be

\footnotetext{
${ }^{1}$ Percent of daily value
}

higher and that of some others be lower than the recommended values. Considering an effective process in or order to standardize home-made formulas and to ensure their nutritional adequacy is essential.

A sample of food stuffs used for a recipe for a homemade formula (28) is demonstrated in Table 5. This composition contains 2019 kilocalorie, $186 \mathrm{~g}$ carbohydrate, $105 \mathrm{~g}$ protein and $95 \mathrm{~g}$ fat. Considering the current price of the foodstuffs, the cost of providing this composition for one day is about 110,000 Rials. On the other hand, the cost of using commercial formulas is 170,000 Rials daily. This difference and the fact that current nutritional services are not covered by insurance may cause patients and their care-givers prefer using a home-made formulas. However, the issues of safety and ease of preparation of commercial formulas should not be ignored. Additionally, in cases of a need for using such especial formulas as elemental formulas, it is very hard, if not impossible, to make it at home.

Overall, the attempts of scientific bodies of clinical nutrition should be towards both improvement of the quality and reduction the costs of commercial formulas and provision guidelines for making efficient home-made formulas. Nonetheless, assuming the cost of one night stay in ICU is averagely 10,000,000 Rials (annually 3,650,000,000 Rials) and the cost of one day use of commercial formulas is 170,000 Rials (annually 62,050,000), if using these formulas can reduce the lenghth of stay in the ICU and overall length of hospitalization even for one night, they can reduce the cost of treatment and save money by 3,587,950,000 Rials annually.

\section{Conclusion}

According to the present study the composition of the commonly available commercial formulas in the Iranian market need to be improved. It is necessary to investigate the effectiveness of the imported and domestic commercial formulas available in the markets using precise clinical trials. Meanwhile, appropriate guidelines for making home-made formulas are also needed.

\section{Limitations}

Some limitations of this study are acknowledged. We did not estimate the costs in relation to the outcomes (efficiency) of home-made and commercial enteral formulas in patients. For instance, we did not analyze the length of hospital stay, costs of care and nosocomial infection rates. Further studies are needed to evaluate and compare outcomes in relation to using different commercial formulas and home-made formulas in the patients.

\section{Acknowledgements}

This study was funded by National Institute of Health Research and Administration of Health Technology Evaluation, Health Deputy, Iran Ministry of Health. MHG was the executive manager. Data were gathered and analyzed by BN. The preliminary paper was written by SM. TRN supervised the whole work and finalized the manuscript. Authors have no conflict of interest to declare. 


\section{Conflict of Interests}

The authors declare that they have no competing interests.

\section{References}

1. Klinkenberg M, Thijs A. Profile of the malnourished patient. Eur J Clin Nutr. 2005;59(10):1129-35

2. McClave SA, Martindale RG, Vanek VW, McCarthy M, Roberts P, Taylor B, et al. Guidelines for the provision and assessment of nutrition support therapy in the adult critically ill patient: Society of Critical Care Medicine (SCCM) and American Society for Parenteral and Enteral Nutrition (ASPEN). J Parenter Enteral Nutr. 2009;33(3):277-316.

3. Berger MM, Shenkin A. Update on clinical micronutrient supplementation studies in the critically ill. Curr Opinion Clin Nutr Metab Care. 2006;9(6):711-6.

4. Giner M, Laviano A, Meguid MM, Gleason JR. In 1995 a correlation between malnutrition and poor outcome in critically ill patients still exists. Nutr. 1996;12(1):23-9.

5. Heyland DK, Dhaliwal R, Drover JW, Gramlich L, Dodek P. Canadian clinical practice guidelines for nutrition support in mechanically ventilated, critically ill adult patients. J Parenter Enteral Nutr. 2003;27(5):355-73

6. Alberda C, Gramlich L, Jones N, Jeejeebhoy K, Day AG, Dhaliwal R, et al. The relationship between nutritional intake and clinical outcomes in critically ill patients: results of an international multicenter observational study. Intensive Care Med. 2009;35(10):1728-37.

7. Heyland DK, Schroter-Noppe D, Drover JW, Jain M, Keefe L, Dhaliwal R, et al. Nutrition support in the critical care setting: current practice in canadian ICUs--opportunities for improvement? J Parenter Enteral Nutr. 2003;27(1):74-83.

8. Visser J, Labadarios D, Blaauw R. Micronutrient supplementation for critically ill adults: a systematic review and meta-analysis. Nutr. 2011;27(7):745-58.

9. Manzanares W, Dhaliwal R, Jiang X, Murch L, Heyland DK. Antioxidant micronutrients in the critically ill: a systematic review and meta-analysis. Crit Care. 2012;16(2):1.

10. Bankhead R, Boullata J, Brantley S, Corkins M, Guenter P, Krenitsky J, et al. Enteral nutrition practice recommendations. J Parenter Enteral Nutr. 2009.

11. White JV, Guenter P, Jensen G, Malone A, Schofield M, Group AMW, et al. Consensus statement of the Academy of Nutrition and Dietetics/American Society for Parenteral and Enteral Nutrition: characteristics recommended for the identification and documentation of adult malnutrition (undernutrition). $\mathrm{J}$ Acad Nutr Diet. 2012;112(5):730-8

12. Lochs H, Dejong C, Hammarqvist F, Hébuterne X, Leon-Sanz M, Schütz $\mathrm{T}$, et al. ESPEN guidelines on enteral nutrition: gastroenterology. Clin Nutr. 2006;25(2):260-74.

13. Loser C, Aschl G, Hebuterne X, Mathus-Vliegen E, Muscaritoli M, Niv $\mathrm{Y}$, et al. ESPEN guidelines on artificial enteral nutritionpercutaneous endoscopic gastrostomy (PEG). Clin Nutr. 2005;24(5):848-61.

14. Arends J, Bodoky G, Bozzetti F, Fearon K, Muscaritoli M, Selga G, et al. ESPEN guidelines on enteral nutrition: non-surgical oncology. Clin Nutr. 2006;25(2):245-59.

15. Kreymann K, Berger M, Deutz Ne, Hiesmayr M, Jolliet P, Kazandjiev G, et al. ESPEN guidelines on enteral nutrition: intensive care. Clin Nutr. 2006;25(2):210-23.

16. Plauth M, Cabre E, Riggio O, Assis-Camilo M, Pirlich M, Kondrup $\mathrm{J}$, et al. ESPEN guidelines on enteral nutrition: liver disease. Clin Nutr. 2006;25(2):285-94.

17. Malone AM. Enteral formula selection: a review of selected product categories. Pract Gastroenterol. 2005;29(6):44.

18. Yokoyama J, Someya Y, Yoshihara R, Ishii H. Effects of highmonounsaturated fatty acid enteral formula versus high-carbohydrate enteral formula on plasma glucose concentration and insulin secretion in healthy individuals and diabetic patients. J Int Med Res. 2008;36(1):137-46

19. Mehta NM, Compher C. ASPEN Clinical Guidelines: nutrition support of the critically ill child. J Parenter Enteral Nutr. 2009;33(3):260-76.

20. Scaife CL, Saffle JR, Morris SE. Intestinal obstruction secondary to enteral feedings in burn trauma patients. J Trauma Acute Care Surg. 1999;47(5):859.

21. Rushdi TA, Pichard C, Khater YH. Control of diarrhea by fiberenriched diet in ICU patients on enteral nutrition: a prospective randomized controlled trial. Clin Nutr. 2004;23(6):1344-52.

22. Sabater-Molina M, Larque E, Torrella F, Zamora S. Dietary fructooligosaccharides and potential benefits on health. J Physiol Biochem. 2009 Sep;65(3):315-28.

23. Preiser J-C, Van Gossum A, Berré J, Vincent J-L, Carpentier Y. Enteral feeding with a solution enriched with antioxidant vitamins A, $\mathrm{C}$, and $\mathrm{E}$ enhances the resistance to oxidative stress. Critic Care Med. 2000;28(12):3828-32

24. Heyland DK, Dhaliwal R, Suchner U, Berger MM. Antioxidant nutrients: a systematic review of trace elements and vitamins in the critically ill patient. Intensive Care Med. 2005;31(3):327-37.

25. Heyland DK, Dhaliwal R, Day AG, Muscedere J, Drover J, Suchner $\mathrm{U}$, et al. Reducing Deaths due to Oxidative Stress (The REDOXSC Study): rationale and study design for a randomized trial of glutamine and antioxidant supplementation in critically-ill patients. Proc Nutr Society. 2006;65(03):250-63.

26. Wang Y, Kang Y, Zhou JR. [Comparison of two types of enteral nutrition in patients with intestinal fistula]. Zhonghua Yi Xue Za Zhi. 2013 Aug 13;93(30):2364-6.

27. Klek S, Hermanowicz A, Dziwiszek G, Matysiak K, Szczepanek K, Szybinski $\mathrm{P}$, et al. Home enteral nutrition reduces complications, length of stay, and health care costs: results from a multicenter study. Am J Clin Nutr. 2014 Aug;100(2):609-15.

28. Iran Nutrition Society Guide for Diet-Therapy (Persian). Tehran: Salemi; 2003 